\title{
Revealing the Surface Energetics and Reactivity of Bimetallic Copper-Gold Catalyst Nanoparticles by In Situ Environmental TEM
}

$\underline{\text { Jaysen Nelayah }}^{1}$, Adrian Chmielewski ${ }^{1}$, Damien Alloyeau ${ }^{1}$, Hakim Amara ${ }^{2}$, Jérôme Creuze ${ }^{3}$ and Christian Ricolleau ${ }^{1}$

1. Université Paris Diderot, Sorbonne Paris Cité, CNRS, Laboratoire Matériaux et Phénomènes Quantiques, UMR 7162, 75013 Paris, France.

2. LEM, Unité Mixte CNRS / ONERA, 29 Av. de la Division Leclerc, 92322 Châtillon, France.

3. ICMMO - UMR 8182 - Bât. 410, Université Paris-Sud 11, 15 rue Georges Clemenceau, 91405 Orsay Cedex, France.

Supported bimetallic copper-gold nanoparticles (NPs) are of interest to heterogeneous catalysis as they often perform better than their monometallic counterparts in many oxidizing and reducing reactions [1]. If the effects of the metal alloying on the catalytic performances of $\mathrm{Cu}-\mathrm{Au}$ NPs are undeniable, the origin of these effects is still largely unknown. This stems from the lack of direct observations of the NPs in their reaction environments, i.e., at high temperature and high pressure. In this contribution, we will present in situ studies of the morphological and chemical transformations of $\mathrm{Au}, \mathrm{Cu}$ and $\mathrm{Cu}-\mathrm{Au}$ nanoparticles at elevated temperature both in vacuum and under $\mathrm{O}_{2}$ and $\mathrm{H}_{2}$ atmospheres using windowed-cell environmental transmission electron microscope (ETEM).

Supported mono- $(\mathrm{Au}, \mathrm{Cu})$ and bimetallic $\mathrm{Cu}-\mathrm{Au}$ NPs were fabricated by pulsed laser deposition [2]. ETEM experiments were performed on a JEOL JEM-ARM200F spherical-aberration-corrected microscope using a Protochips gas cell. In the first part, we will present an original approach based on bulk plasmon nanothermometry using aluminum nanospheres to determine local temperatures in a gas cell under operation [3]. With access to local temperature under operating conditions, we will assess the thermal homogeneity and stability across the heater region of our Protochips gas cell and the effects of increasing gas pressure on the local temperature.

Then, we will discuss the thermal stability of SiN-supported $\mathrm{Au}-\mathrm{Cu}$ NPs heated in vacuum up to $800{ }^{\circ} \mathrm{C}$. In particular, we will present a robust methodology to experimentally determine the absolute surface energy of bimetallic nanoalloys using in situ TEM imaging. This methodology relies on the real-time monitoring of the evaporation of bimetallic NPs in vacuum and the extension of the Kelvin equation to two-component systems. In $\mathrm{Cu}$-Au nanoalloys in liquid state, the determination of the absolute values of the surface energy suggests that their surface energy follows a Vegard's law-like behavior. These experimental observations are confirmed by Monte Carlo simulations which bring additional insights into the structural stability and thermodynamic properties of $\mathrm{Cu}-\mathrm{Au}$ NPs both in solid and liquid states [4].

The final part will focus on sintering dynamics and structural evolution of $\mathrm{TiO}_{2}$-supported $\mathrm{Au}, \mathrm{Cu}$ and $\mathrm{Cu}-\mathrm{Au}$ nanoparticles under oxidizing $\mathrm{O}_{2}$ and $\mathrm{H}_{2}$ atmospheres up to atmospheric pressure and temperatures up to $600{ }^{\circ} \mathrm{C}$. Figure 2a shows an HAADF-STEM image of a $\mathrm{Cu}$ NP under $0.5 \mathrm{~atm}$ of $\mathrm{O}_{2}$ at $400{ }^{\circ} \mathrm{C}$. In these environments, the initial metallic structure $\left(\mathrm{Cu}^{0}\right)$ of the NP is fully oxidized to a cuprite structure $\left(\mathrm{Cu}_{2} \mathrm{O}\right)$. The resulting morphology is a truncated octahedron, bounded by (111) and (100) facets. Figure $2 \mathrm{~b}$ shows the same particle under $0.5 \mathrm{~atm}$ of $\mathrm{H}_{2}$ at $400^{\circ} \mathrm{C}$. Under the reductive atmosphere, the cuprite structure remains stable. However, one can observe that the extension (111) and (100) facets have slightly changed. This implies a change in surface energy of these two facets, induced by the change of the reaction medium. Similar temperature- and gasinduced structural changes have been observed in $\mathrm{Au}$ and $\mathrm{Cu}-\mathrm{Au}$ NPs. These observations will be presented and discussed to highlight the effect of alloying gold and copper on their respective structural stability under reaction conditions. [5] 


\section{References:}

[1] CL Bracey et al, Chem Soc Rev 38 (2009), p. 2231.

[2] H Prunier et al, Phys Chem Chem Phys 17 (2015), 28339.

[3] A Chmielewski et al, in preparation.

[4] A Chmielewski et al, Phys Rev Lett 120 (2018), 025901.

[5] The authors acknowledge funding from the French National Research Agency through the TOTEM project, Grant Number ANR-17-CE07-0031.
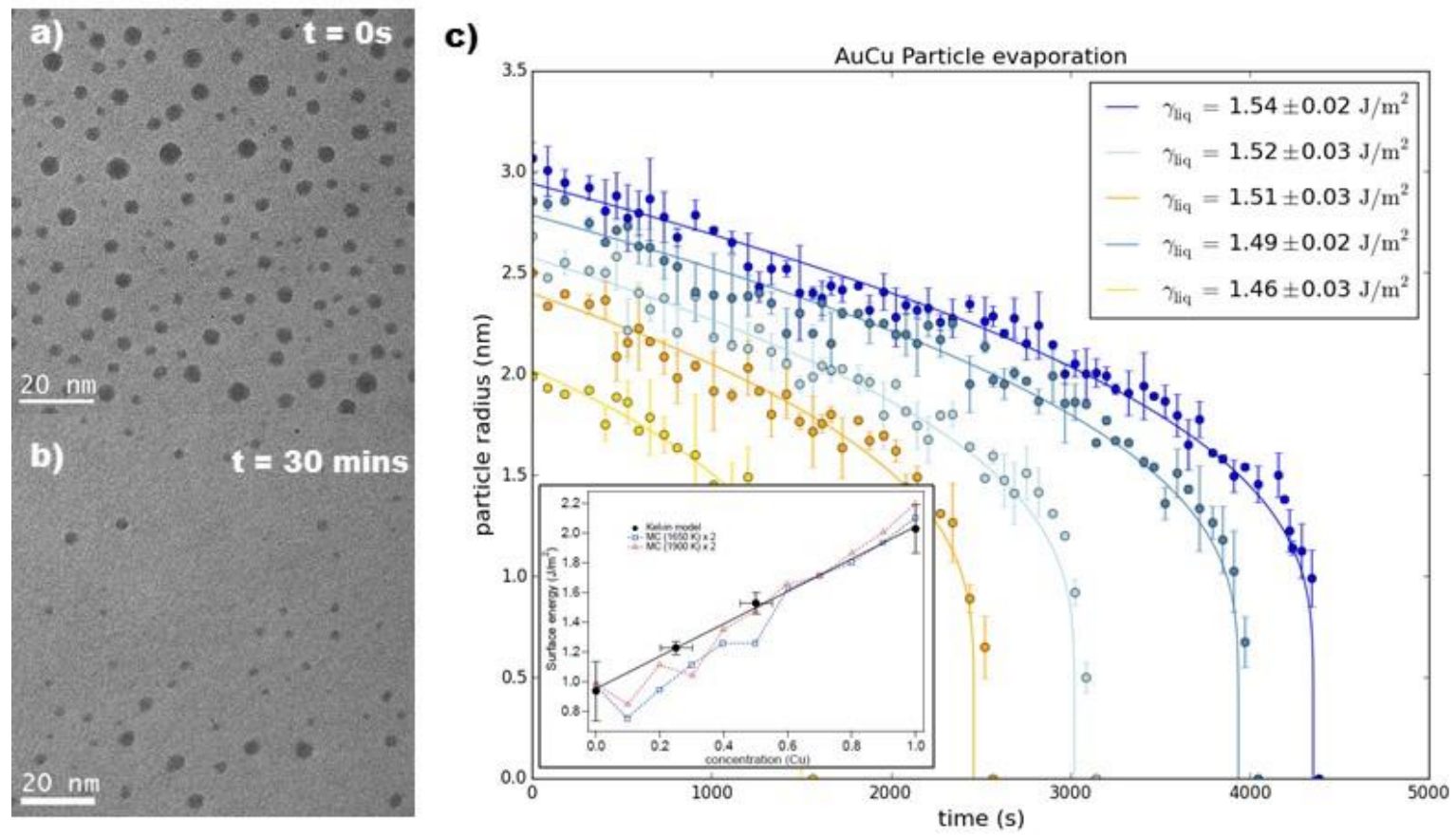

Figure 1. TEM images of the evolution of CuAu NPs at $700{ }^{\circ} \mathrm{C}$ at (a) $t=0 \mathrm{~s}$ and (b) $\mathrm{t}=30$ mins. (c)Variation of the radii of six CuAu NPs with time. Insets: corresponding extracted surface energies (right), variation of the surface energy of $\mathrm{Cu}-\mathrm{Au}$ NPs in liquid state with comosition, blue-red lines are Monte Carlo simulations, the full black line is a linear fit of experimental data (left).
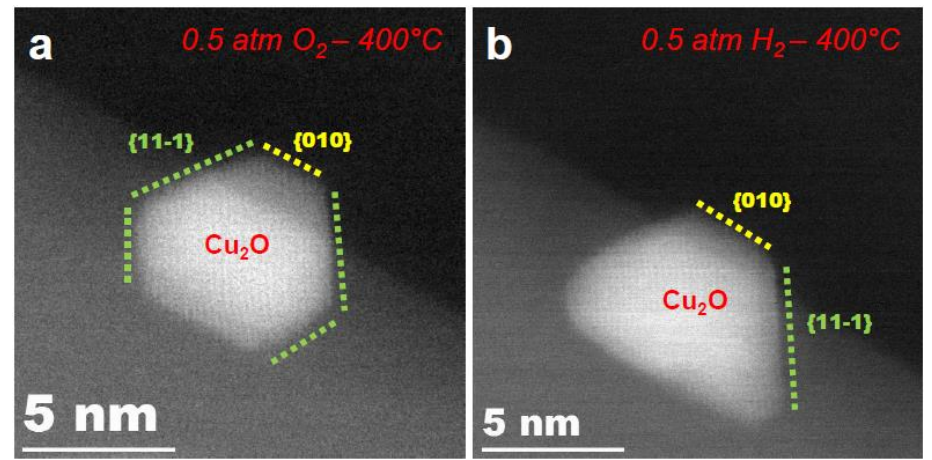

Figure 2. HAADF-STEM images of a rutile- $\mathrm{TiO}_{2}$ supported $\mathrm{Cu}$ nanoparticle under $0.5 \mathrm{~atm}$ of (a) $\mathrm{O}_{2}$ and (b) $\mathrm{H}_{2}$ gases at $400^{\circ} \mathrm{C}$. 